\title{
Supervised Learning for Decorrelated Gaussian Networks
}

\author{
D. Obradovic and G. Deco \\ Siemens AG, Corporate R\&D, Otto-Hahn-Ring 6, 8000 Munich 83, Germany
}

This paper presents a new two-stage leaming paradigm that utilizes localization properties of Gaussian neurons. In the first stage, a single layer of Gaussian function is trained in a novel unsupervised fashion to model the distribution of the network input. The input model is obtained by minimizing a cost function whose first term can be seen as an implementation of the standard Hebbian leaming law. The second term of the cost function has an "anti-Hebbian" effect which reinforces the competitive learning. In the second stage of the learning paradigm, the previously obtained receptive field distribution is further used for function approximation. For comparison, a standard single hidden-layer Gaussian network is optimized with the initial centers corresponding to the first stage learning.

Let a layer of Gaussian-neurons with centers $w_{i}$ and widths $\sigma_{i}$ has normalized outputs given by $O_{i}(\xi)$,where $\xi$ is the input vector. Furthermore, let us define a cost function $H^{\prime}{ }_{i}$ as follows:

$$
H^{\prime}=-\sum_{i}\left(\int d \xi P(\xi) O_{i}(\xi)\right)+\alpha \sum_{i} \sum_{j \neq i} \int d \xi P(\xi) O_{i}(\xi) O_{j}(\xi)
$$

where $P(\xi)$ is the probability density of the input patterns. Unsupervised learning is defined as minimization of the cost function presented in equation (EQ 1) with respect to the centers $w_{i}$. The first term on the r.h.s. of (EQ 1) induces attraction of all neurons toward the region of the input space were input data exist. The second term of the cost function penalizes overlapping of the outputs of the neurons with respect to each pattem. We refer to this learning procedure as the Decorrelated Hebbian Leaming (DHL). We have shown that the value of scaling factor $\alpha$ is irrelevant due to the normalization of the Gaussian outputs.

Once when the centers of the Gaussians are determined, the output of the neurons are used to obtain a weighted piece-wise linear approximation of the single-output function by minimizing the following costfunction:

$$
J=\frac{1}{N} \sum_{j=1}^{N}\left\{z_{j}-\sum_{i=1}^{m}\left[a_{i}+\xi_{j} b_{i}\right] O_{i}\left(\xi_{j}\right)\right\}^{2}=\frac{1}{N} \sum_{j=1}^{N}\left(\text { error }_{j}\right)^{2}
$$

where $z_{j}$ is the " $\mathrm{j}$-th" pattem of the function output, $N$ is the number of patterns and $m$ is the number of the Gaussian neurons. The parameters to be adjusted are $a_{i}$ and the vector $b_{i}$ whose dimension is equal to the number of function inputs. Hence, the total number of parameters to be determined in the second learning stage is $m^{*}[1+$ (number of function inputs)].

As an example we use the standard benchmark Mackey-Glass time series. The DHL is used as unsupervised leaming method for positioning the centers of the Gaussians neurons to fit the Mackey-Glass chaotic attractor. The test set consists of 500 points not contained in the training set of 1000 data points. Once when the centers are determined, a piece-wise linear approximation of the function is obtained by minimizing the cost function defined in (EQ 2).

\begin{tabular}{||c||c||c||}
\hline Approximation Type & Number of Parameters & Normalized Error \\
\hline $\begin{array}{c}\text { DHL + Optimal Linear } \\
\text { Output Layer }\end{array}$ & $401+100$ & 0.2127 \\
\hline \hline $\begin{array}{c}\text { DHL + Piece-Wise Linear } \\
\text { Approximation }\end{array}$ & $401+500$ & 0.056 \\
\hline \hline $\begin{array}{c}\text { Quasi-Newton } \\
\text { (DHL for initialization) }\end{array}$ & 901 & 0.0376 \\
\hline \hline Locally Tuned Gaussian & 900 & 0.23 \\
\hline
\end{tabular}

